Article

\title{
Acacetin Protects Mice from Staphylococcus aureus Bloodstream Infection by Inhibiting the Activity of Sortase A
}

\author{
Chongwei Bi ${ }^{1,+}$, Xiaoyun Dong ${ }^{2,+}$, Xiaobo Zhong ${ }^{1}$, Hongjun Cai ${ }^{3}$, Dacheng Wang ${ }^{1, *}$ \\ and Lin Wang ${ }^{4, *}$ \\ 1 College of Animal Science, Jilin University, Changchun 130062, China; bicwei@gmail.com (C.B.); \\ 15043029208@163.com (X.Z.) \\ 2 Department of Pharmacology, College of Basic Medical Science, Jilin University, Changchun 130062, China; \\ banyuexiao@126.com \\ 3 College of Animal Science and Technology, Jilin Agricultural University, Changchun 130062, China; \\ chj6788571@163.com \\ 4 Key Laboratory of Zoonosis Research, Ministry of Education/Institute of Zoonosis/College of Veterinary \\ Medicine, Jilin University, Changchun 130062, China \\ * Correspondence: wangdc@jlu.edu.cn (D.W.); wanglin1020@jlu.edu.cn (L.W.); \\ Tel.: +86-431-8783-5385 (L.W.); Fax: +86-431-8783-6160 (L.W.) \\ + These authors contributed equally to this work. \\ Academic Editor: Diego Muñoz-Torrero \\ Received: 16 August 2016; Accepted: 22 September 2016; Published: 26 September 2016
}

\begin{abstract}
Staphylococcus aureus (S. aureus) is a major cause of infection in hospitals and communities. Widespread dissemination of multi-drug resistant $S$. aureus is a serious threat to the health of humans and animals. An anti-virulence strategy has been widely considered as an alternative therapeutic approach. Inhibitors of virulence factors are able to treat $S$. aureus infections without influencing the growth or viability of bacteria and rarely lead to bacterial resistance. Sortase A (SrtA) is a membrane-associated cysteine transpeptidase that catalyzes up to 25 surface proteins that covalently bind to cell wall peptidoglycans. In $S$. aureus, most of these surface proteins have been identified as important virulence factors that are vital in bacterial pathogenesis. In the present study, we show that acacetin, a natural flavonoid compound, inhibits the activity of SrtA in S. aureus $\left(\mathrm{IC}_{50}=36.46 \pm 4.69 \mu \mathrm{g} / \mathrm{mL}, 128 \mu \mathrm{M}\right)$ which affects the assembly of protein A (SpA) to cell walls and reduces the binding of $S$. aureus to fibrinogen $(\mathrm{Fg})$. The mechanism of the interaction between acacetin and SrtA were preliminarily discussed using molecular dynamics simulations. The results suggested that acacetin adopted a compact conformation binding at the pocket of the SrtA via residues Arg-139 and Lys-140. By performing an animal infection model, we demonstrated that acacetin was able to protect mice from renal abscess formation induced by $S$. aureus and significantly increased survival rates. Taken together, these findings suggest that acacetin may be a promising candidate for the development of anti-S. aureus drugs.
\end{abstract}

Keywords: Staphylococcus aureus; sortase A; acacetin; inhibitor; renal abscess

\section{Introduction}

Anti-virulence strategies have been proposed recently to combat the increasing drug resistance caused by conventional antibiotics combined with a salient lack of research and development of new antibiotics to counteract bacterial pathogens [1]. Many surface proteins of Gram-positive bacteria are thought to be important virulence factors because these surface proteins play key roles in multiple aspects of pathogenesis, such as immune evasion, adhesion and invasion of host cells [2-4]. 
These proteins are covalently linked to the peptidoglycan molecules that compose the cell wall by sortase enzymes [5,6].

SrtA from S. aureus (Sa-SrtA) has been extensively studied since its discovery in 1999 [7,8]. Several well-proven virulence factors are wall anchored by Sa-SrtA, including SpA, the fibrinogen-binding clumping factors $\mathrm{ClfA}$ and $\mathrm{ClfB}$, and the fibronectin-binding proteins FnbA and FnbB $[9,10]$. Additionally, SrtA is not essential for the growth and viability of bacteria. Therefore, inhibiting SrtA would be less likely to induce selection pressure, thereby reducing the possibility of resistance developing. In addition, there are no SrtA homologues in eukaryotes with closest function; therefore, inhibiting SrtA is unlikely to result in particularly severe side effects [11,12]. These characteristic features make SrtA a potential target for the treatment of $S$. aureus infection with promising pharmaceutical prospects.

Earlier work identified srtA mutants that failed to anchor proteins to the cell wall peptidoglycans [13], displayed great attenuation of virulence and were incapable of leading to sepsis or the formation of abscesses in staphylococcal-infected mice models [9,14-16]. Subsequent to the discovery above, the search for and development of SrtA inhibitors has been a hot spot in the research into antimicrobial agents, and several candidates have been investigated [17-19].

Several strategies have been developed for the identification and characterization of new SrtA inhibitors. The most widely used strategies include screening of natural product or small compound libraries based on enzyme activity, screening based on molecular modelling, pharmacophore hypotheses, 3D-QSAR models and virtual screening, which led to the discovery of natural and chemically synthesized products (reviewed in [17]). In a previous study in our lab, the natural products quercitrin [20] and chlorogenic acid [21] were found to be potent SrtA inhibitors ( $\mathrm{IC}_{50}=72 \mu \mathrm{M}$ and $96 \mu \mathrm{M}$, respectively). Accordingly, this paper aimed to enlarge the screening range, select a new natural compound with anti-virulence capacity in vivo and in vitro, clarify the mechanisms, and provide a new lead compound or drug candidate for the treatment of $S$. aureus infection.

By performing the FRET assay, acacetin emerged as the best candidate among 50 types of natural compounds with diverse structures that we screened. Acacetin (Figure 1A; IUPAC name: 5,7-dihydroxy-2-(4-methoxyphenyl)chromen-4-one) is an O-methylated flavone which is found in Robinia pseudoacacia, Chrysanthemi indici Flos, Tunera diffusa and Betula pendula [22,23].

A<smiles>COc1ccc(-c2cc(=O)c3c(O)cc(O)cc3o2)cc1</smiles>

B

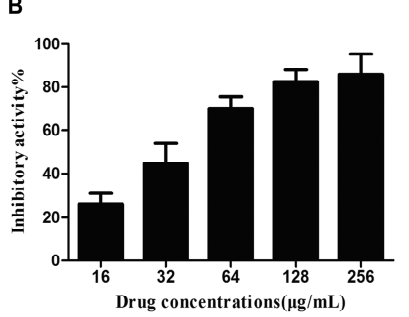

C

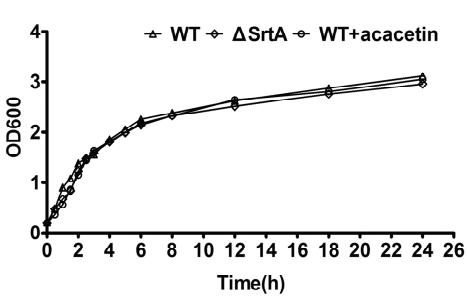

Figure 1. (A) Structure of acacetin; (B) Inhibitory activity of acacetin (gradient concentrations) against S. aureus SrtA in vitro; (C) Growth curves of S. aureus ATCC25904 (WT) with or without acacetin $(256 \mu \mathrm{g} / \mathrm{mL})$ addition and $\Delta$ SrtA.

Acacetin exhibits outstanding anti-peroxidative and anticancer activities and is effective on various cancer cell lines, including lung, leukaemia, prostate, and breast cancer [24-27]. The anti-arthritic and anti-inflammatory effects of acacetin have also been confirmed in vitro [28]. In particular, a recent research reported that acacetin isolated from the leaves of Combretum vendae 
was active against $S$. aureus [29]. In the present study, we evaluated the anti-S. aureus efficacy of acacetin in inhibiting the activity of SrtA by using well-established models in vitro, ex-vivo and in vivo. We further investigated the interaction mechanism of acacetin-SrtA complex using a molecular dynamics simulation study.

\section{Results}

\subsection{Acacetin Inhibits SrtA-Catalyzed Transpeptidation}

The inhibitory activity of acacetin against SrtA was determined using a FRET method [13,30]. A fluorescent peptide substrate (Dabcyl-QALPETGEE-Edans) of SrtA was constructed to monitor the change of the fluorescence during SrtA catalysis. The results were calculated as the inhibition rate, which was expressed as a percentage. Among the tested compounds, we found that acacetin, which is a natural bioflavonoid, had significant catalytic inhibition on SrtA. The detailed results show that the activity of SrtA was blocked by acacetin in a dose-dependent manner (Figure 1B). Based on the inhibition rate of diverse inhibitor concentrations, we calculated an $\mathrm{IC}_{50}$ of $36.46 \pm 4.69 \mu \mathrm{g} / \mathrm{mL} \mathrm{for}$ acacetin in the inhibition of SrtA.

\subsection{Acacetin Has No Influence on the Growth of S. aureus}

Preliminary experiments showed that the MIC values of acacetin on S. aureus (ATCC25904 and $\Delta$ SrtA strain) were both greater than $1024 \mu \mathrm{g} / \mathrm{mL}$. Further, a growth curve was studied to reveal whether the growth states varied after the addition of acacetin. According to the results, the growth rate of $S$. aureus WT and $\Delta$ SrtA strain was analogous to $\mathrm{WT}+$ acacetin $(256 \mu \mathrm{g} / \mathrm{mL})$, even when the concentration was seven times its $\mathrm{IC}_{50}$ (Figure $1 \mathrm{C}$ ). This result indicates that acacetin was able to effectively reduce the activity of SrtA at a dosage that was much lower than its MIC.

\subsection{Acacetin Influences the Assembly of SpA into the Cell Wall}

In S. aureus, SrtA anchors more than 20 surface proteins to cell walls, including SpA, which is a multifunctional surface protein that binds to the Fc $\gamma$ portion of IgG, which in turn is important for immune evasion. Based on its function, we investigated the content variation of SpA in cell wall after the addition of acacetin by staining Staphylococci with FITC-labelled goat anti-rabbit IgG. The images obtained from confocal laser-scanning microscope show that S. aureus ATCC25904 bound FITC-labelled IgG, which resulted in cells that were covered with an obvious green colour (Figure 2). In contrast, the $\Delta$ SrtA strain that lacked SrtA function failed to bind the fluorescent substance. The green fluorescence was significantly weaker when acacetin $(256 \mu \mathrm{g} / \mathrm{mL})$ was added to the broth. These results collectively suggested that acacetin was able to disturb the assembly of SpA into the cell wall by inhibiting SrtA activity.

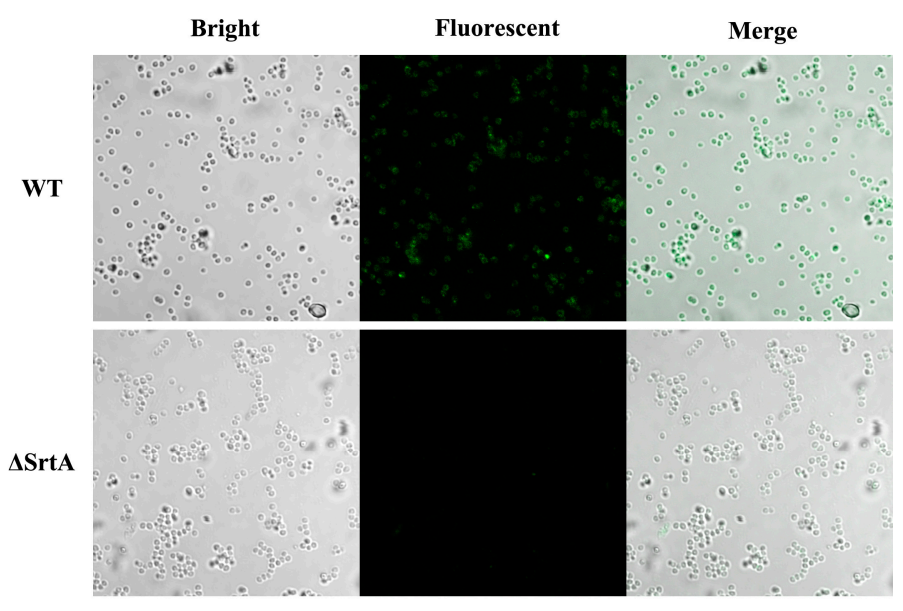

Figure 2. Cont. 


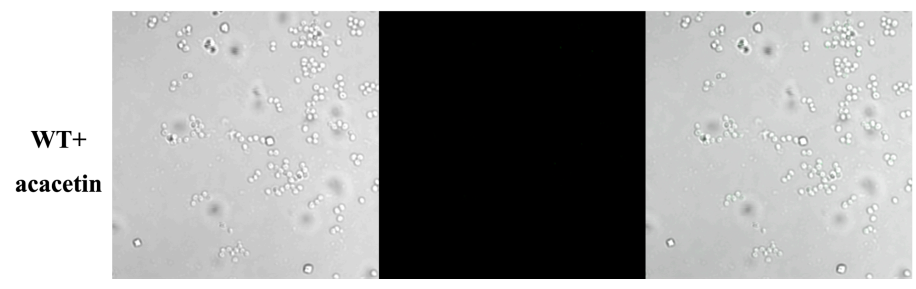

Figure 2. Effects of acacetin on the display of SpA on the surface of S. aureus ATCC25904. Binding of FITC-labeled Ig to SpA was viewed via confocal laser-scanning microscope. WT S. aureus cells were covered with an obvious green colour. $\Delta$ SrtA failed to bind FITC-labeled Ig; the green fluorescence was significantly weaker after acacetin $(256 \mu \mathrm{g} / \mathrm{mL})$ addition.

\subsection{Acacetin Reduces the Adherence of S. aureus to Fibrinogen}

Previous conclusions illustrated that acacetin was able to significantly inhibit the SrtA-catalyzed transpeptidation at sub-MIC concentrations. S. aureus expresses up to 25 types of surface proteins that are anchored by SrtA, most of which are essential in the pathogenesis of staphylococcal infection. These surface proteins include clumping factors (ClfA and ClfB), which are vital for the attachment of Staphylococci to host Fg, which is the initiation of infection. A virulence reduction of SrtA-deficient S. aureus was found in a previous study [9]. One important reason might be that the mutants that lacked SrtA failed to bind Clfs to cell walls, resulting in the loss of Fg-binding function. To test this hypothesis, we performed a Fg-binding assay. The adhesion results showed that the $\Delta$ SrtA strain had a minimum binding rate to $\mathrm{Fg}(6.02 \pm 2.09 \%)$ (Figure 3), indicating that the Fg-binding function of the $\Delta$ SrtA strain was almost completely lost. Treating ATCC25904 (WT) with acacetin significantly reduced the adhesion rates in a concentration-dependent manner compared to no addition (WT). When $32(112 \mu \mathrm{M}), 64(224 \mu \mathrm{M}), 128(448 \mu \mathrm{M})$, or $256(896 \mu \mathrm{M}) \mu \mathrm{g} / \mathrm{mL}$ of acacetin was added, the adhesion rates were $83.35 \pm 5.15 \%, 55.33 \pm 5.12 \%, 30.17 \pm 4.7 \%$, and $15.77 \pm 3.81 \%$, respectively (Figure 2 ).

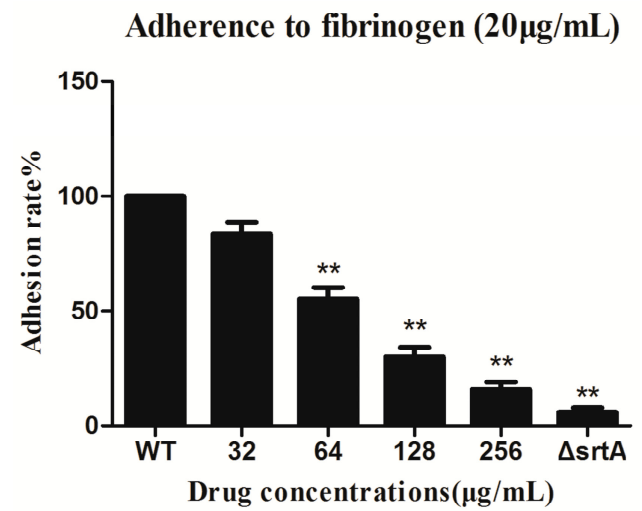

Figure 3. Adhesion rate of S. aureus to Fg. Adhesion was significantly inhibited by acacetin in a dose dependent manner. The value of the $\Delta$ SrtA was used as a positive control. Three independent experiments were taken to obtain stable result. $* *$ represents $p<0.01 \mathrm{vs}$. the WT group.

\subsection{Determination of the SrtA-Acacetin Binding Mechanism}

SrtA-acacetin complex was equilibrated after 20 ns MD simulation, and the plot of root-mean-square deviation (RMSD) (in Ångstroms) of the complex is shown to determine that the system is stabilized during the simulation in Figure 4A. The theoretical binding mode of the acacetin and SrtA is shown in Figure 4B,C. Acacetin adopted a compact conformation binding at the pocket area of SrtA (Figure 4B) and blocked the passway of substrate into the active site. The phenyl group of acacetin was positioned at the hydrophobic pocket, surrounded by the residues Ala-34, Pro-36, Leu-39, Ala-60, Gly-61 and Trp-136 (Figure 4C). Detailed analysis showed that the phenyl group of acacetin 
formed a $\mathrm{CH}-\pi$ interaction with residue Trp-136. Importantly, the carbonyl " $O$ " at the 4-position and the hydroxyl group at the 7-position of acacetin formed two hydrogen bond interactions with the residues Arg-139 (bond length: 2.6 $\AA$ ) and Lys-140 (bond length: $1.8 \AA$ ), respectively, which were the main interactions between acacetin and SrtA. In summary, the above molecular dynamics simulation gave us a rational explanation of the interactions between acacetin and SrtA, which provided valuable information for the further development of SrtA inhibitors.

A

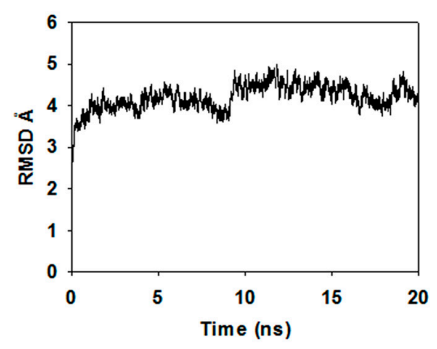

B

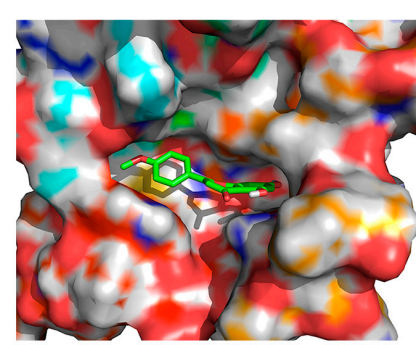

C

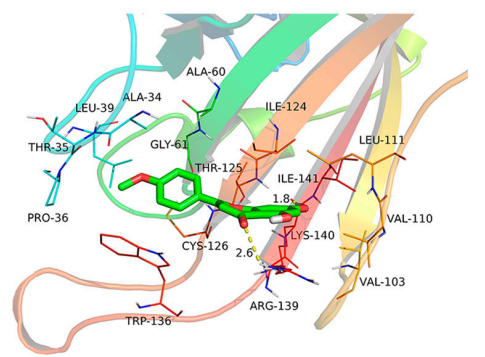

Figure 4. (A) Plot of RMSD (in $\AA$ ) for the SrtA-acacetin complex during $20 \mathrm{~ns}$ MD simulation; (B) The binding model of SrtA-acacetin complex was shown by PyMoL [http://www.pymol.org/]. The solvent-accessible surface of the protein and the structure of the bound small molecule compound are shown. The surface has been colored to indicate the electrostatic properties from acidic (red) to basic (blue); (C) Ribbon diagram of SrtA-acacetin complex was shown by PyMoL.

\subsection{Acacetin Significantly Increases the Survival Rate of S. aureus-Infected Mice}

To investigate the protective effects of acacetin on S. aureus-infected mice in vivo, we conducted both fatal and non-fatal infections in the present study. Six days after injection with $2 \times 10^{9} \mathrm{CFU}$ of S. aureus, which is a lethal concentration for animals, $90 \%$ of the mice infected with the WT strain had died. In contrast to the WT strain, the mortality rate of $\Delta$ SrtA was still $0 \%$ by the observation deadline (Figure 5). The data indicated that SrtA is a considerable virulence factor of $S$. aureus-induced lethal infection. A potential inhibitor of SrtA should have similar performance to become a powerful therapeutic candidate. Drug-treated mice received hypodermic injections of acacetin $(150 \mathrm{mg} / \mathrm{kg} / \mathrm{d})$ after initial infection and then died of $S$. aureus bacteremia five days after infection. The detailed results suggested that the acacetin-treated mice experienced a significant survival advantage compared to the WT group (Figure 5).

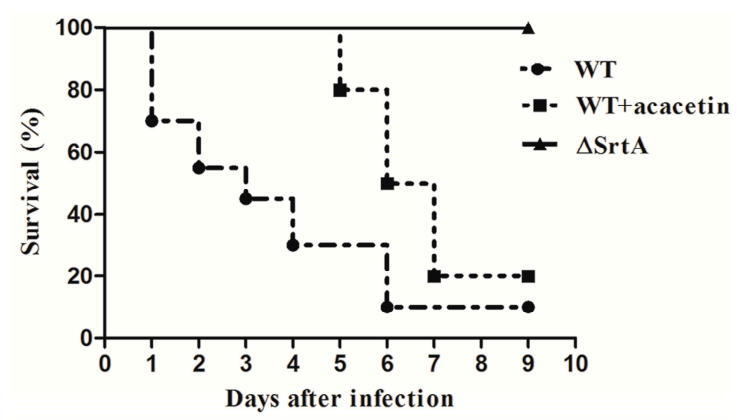

Figure 5. Survival rates of $S$. aureus-infected BALB/c mice. Mice $(N=20)$ were monitored for 9 days after tail vein injection of $2 \times 10^{9} \mathrm{CFU}$ of $S$. aureus (WT) and $\triangle \mathrm{SrtA}$. Treatment with acacetin (150 mg/kg/d) was taken after initial infection. Log-rank (Mantel-Cox) test was used for survival statistics. The statistical significance determined as follows: WT vs. WT + acacetin, $p<0.05$; WT vs. $\Delta$ SrtA, $p<0.0001$.

The survival rates were still above $20 \%$ on the ninth day after infection. These data indicated that acacetin can significantly prolong the survival of mice with blood-borne infections. 


\subsection{Acacetin Alleviates the Symptoms of S. aureus-Induced Renal Abscess in Mice}

Bloodstream-infected S. aureus typically attaches to specific tissues after evading innate immune defences and then induces the formation of organ abscesses [31]. Previous research suggested that the various surface proteins that are catalyzed by SrtA are vital in S. aureus pathogenesis, including SpA, FnbpA and ClfA [14]. By performing survival experiments, we illustrated that acacetin can effectively protect mice from fatal S. aureus infection. To study the effect of acacetin on non-fatal S. aureus-induced infection, we performed a renal abscess formation experiment by i.v. injecting mice with $2 \times 10^{9} \mathrm{CFU}$ of S. aureus. In the drug-treated group, acacetin $(150 \mathrm{mg} / \mathrm{kg} / \mathrm{d})$ was first hypodermically injected $30 \mathrm{~min}$ after the initial S. aureus infection. Six days after the injection, the mice were euthanized and dissected to excise the kidneys. The left kidneys of the infected mice were homogenized, distributed in PBS, and then plated for the enumeration of CFUs. The CFUs results show that the WT group exhibited a mean of $6.2 \times 10^{5} \mathrm{CFU} / \mathrm{g}$ in the kidneys, which is remarkably larger than in the $\Delta \operatorname{SrtA}$ group $(11.6 \mathrm{CFU} / \mathrm{g})$ and acacetin-treated group $\left(10^{3} \mathrm{CFU} / \mathrm{g}\right)$ (Figure $\left.6 \mathrm{P}\right)$. The right kidneys were fixed with formalin solution $(10 \%)$ for histopathology analysis. In macroscopic view, we observed a significant abscess forming on the surface of the WT kidney (Figure 6A), which was larger than that of the acacetin-treated group (Figure 6C). The $\Delta$ SrtA kidneys failed to manifest abscess formation and exhibited a normal size and shape (Figure 6B). The results of the pathological section are consistent with the kidney photos. A large area of $S$. aureus is surrounded by polymorphonuclear leukocytes (PMNs), amorphous material and eosinophils in kidneys from WT challenged mice (Figure 6D,E,J,K) [14]. Mice treated with acacetin were prominently alleviated from the symptoms of renal abscess, resulting in a smaller abscess size and fewer PMNs and Staphylococci (Figure 6H,I,N,O). These findings suggest that acacetin can protect mice from $S$. aureus-induced infection and reduce the formation of renal abscesses.

\section{Discussion}

S. aureus is notorious as a major source of hospital- and community-acquired infections [32]. Bacterial resistance to traditional antibiotics is increasing and becoming more broad-spectrum, leading to the widespread distribution of drug-resistant strains; as a result, the treatment of $S$. aureus infection is becoming increasingly difficult [33]. Anti-virulence strategies have aroused wide interest as alternative approaches for the prevention and treatment of bacterial infections. Because virulence factors are not indispensable for bacterial growth, this strategy might avoid the development of drug resistance.

We have screened potent SrtA inhibitors from various types of natural compounds utilizing the FRET-based cleavage of LPETG as a measure of sortase activity. Acacetin, an O-methylated flavone, was able to significantly inhibit SrtA activity both in vitro and ex-vivo without showing a noticeable antimicrobial effect. To our knowledge, only a few articles have reported in vivo therapeutic effects of SrtA inhibitors and validated their inhibitory activity in vitro [34,35]. Although we have reported in detail the activity of chlorogenic acid as an inhibitor of SrtA in vitro and in vivo [21], the flavonoid acacetin, in terms of its structure and mechanisms of interaction with SrtA, is very different. In addition, to date, there are almost no related reports about the antibacterial activity of acacetin, apart from a recent study on acacetin isolated from the leaves of Combretum vendae [29]. However, our results indicated that acacetin was able to effectively inhibit the SrtA-catalyzed transpeptidation at sub-MIC concentrations.

We also investigated the protective effects of acacetin on S. aureus-infected mice in vivo. The results suggested that acacetin was able to prolong the survival of mice and alleviate the symptoms of renal abscesses (Figures 5 and 6). Molecular docking and molecular simulation were also used in this study to reveal the binding mechanism of acacetin with SrtA. The results indicated that the main interactions between acacetin and SrtA were the carbonyl " $O$ " at the 4-position and the hydroxyl group at the 7-position of acacetin, which formed two hydrogen bond interactions with the residues Arg-139 and Lys-140, respectively (Figure 4). 

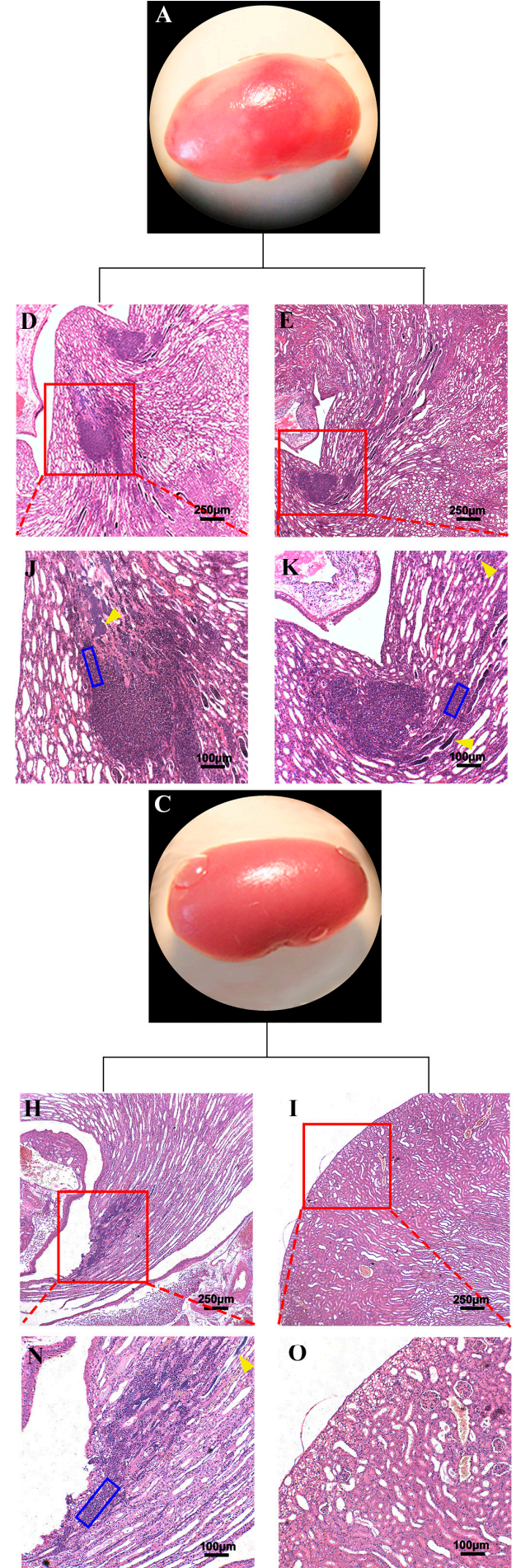
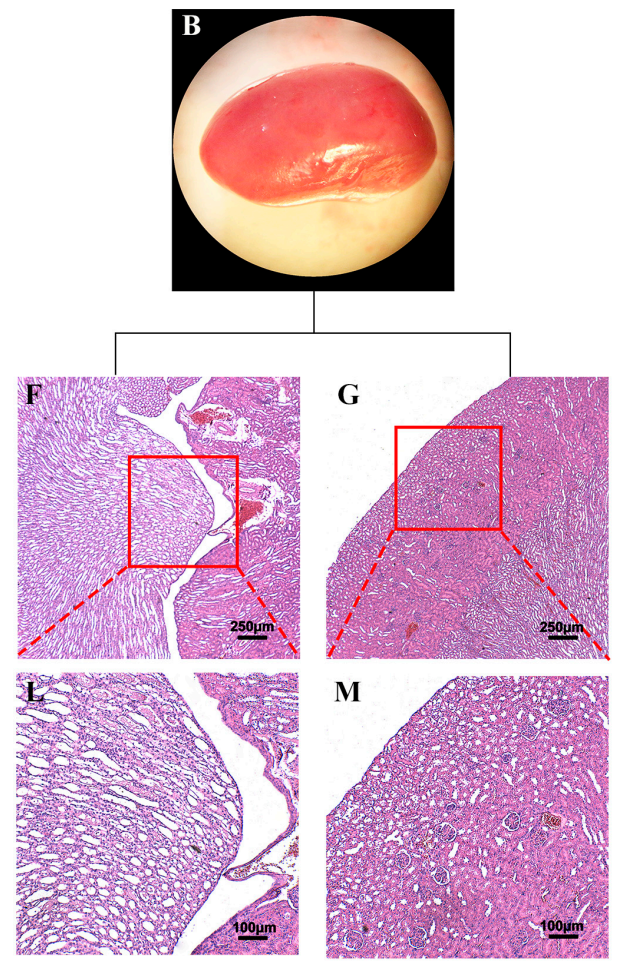

P

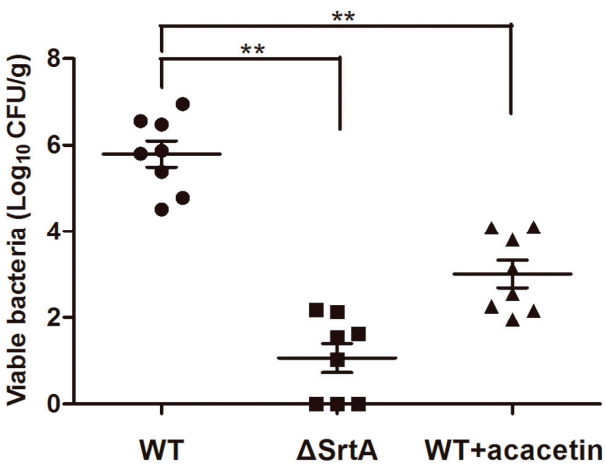

Figure 6. Effects of acacetin on S. aureus-induced abscess formation in kidney of BALB/c mice. BALB/c mice ( $\mathrm{N}=8$ per group) was infected with $2 \times 10^{8} \mathrm{CFU}$ of $S$. aureus ATCC25904 (WT), $\triangle$ SrtA or WT treated with acacetin $(150 \mathrm{mg} / \mathrm{kg} / \mathrm{d})$. (A-O) Kidneys were excised for observing surface abscesses (A-C) or stained with H\&E. Histopathology images were acquired with light microscopy at $\times 100$ (D-I) and $\times 400(\mathbf{J}-\mathbf{O})$. WT infected kidneys exhibited an enclosed Staphylococci in the central area (J,K, yellow arrowheads), surrounded by a large cuff of PMNs (J,K, blue rectangular box). Acacetin-treated kidneys only had a few Staphylococci (H, yellow arrowheads) and some slight influxes of PMNs (H, blue rectangular box). (P) Bacterial burden were obtained from homogenized renal tissues on the 7th day after infection and calculated as $\log _{10} \mathrm{CFU} / \mathrm{g}$. Student's $t$-test was performed to determine the statistical significance; $* *$ represents $p<0.01$ vs. the WT group. 


\section{Materials and Methods}

\subsection{Microbial Strains, Plasmids and Reagents}

The reference strains S. aureus ATCC25904 (SrtA positive; ClfA positive; coagulase negative; non-haemolytic) was used in this work. The $\Delta$ SrtA strain was constructed using ATCC25904 in our previous work [36]. The SrtA expression vector pGSrtA $\mathrm{PN}_{\Delta \mathrm{N} 9}$ was obtained by cloning the SrtA $\mathrm{A}_{\Delta \mathrm{N} 59}$ sequence into a pGEX-6P-1 vector. S. aureus ATCC25904 and $\Delta$ SrtA strains were cultured in brain-heart infusion (BHI) solution (Oxoid, Basingstoke, UK) at $37{ }^{\circ} \mathrm{C}$ with shaking at $220 \mathrm{rpm}$. The $\mathrm{SrtA}_{\Delta \mathrm{N} 59}$ protein was expressed from $\mathrm{pGSrtA}_{\Delta \mathrm{N} 59}$ vector transformed E. coli strain BL21 (DE3) and purified by GST affinity chromatography column. Fluorescent-quenched peptide substrate Dabcyl-QALPETGEE-Edans was purchased from Shanghai GL Biochem (Shanghai, China). The acacetin compound was obtained from Chengdu Pufeide Biotech Company (Chengdu, China).

\subsection{Inhibition of Sortase A Activity}

Sortase A activity inhibition assay was performed in 96-well black plates. Fluorescent peptide substrate Dabcyl-QALPETGEE-Edans was used to determine the $\mathrm{IC}_{50}$ value. This assay was based on a fluorescence resonance energy transfer (FRET) method that has been described previously [13,30]. Briefly, the reactions contained $10 \mu \mathrm{M}$ of peptide substrate, $4 \mu \mathrm{M}$ of Srt $\mathrm{A}_{\Delta \mathrm{N} 59}$ protein and the assay buffer $(150 \mathrm{mM} \mathrm{NaCl}, 5 \mathrm{mM} \mathrm{CaCl} 2,0.1 \%$ Triton X-100 and $50 \mathrm{mM}$ Tris- $\mathrm{HCl}, \mathrm{pH} 7.5)$ in a final volume of $300 \mu \mathrm{L}$. Increasing concentrations of acacetin and SrtA $\mathrm{AN}_{59}$ protein were added to the plate and incubated at $37^{\circ} \mathrm{C}$ for $1 \mathrm{~h}$; the peptide substrate was then added, and the reaction proceeded at $37^{\circ} \mathrm{C}$ for $1 \mathrm{~h}$. The increase in fluorescence intensity was measured using a microplate reader (Infinite ${ }^{\circledR}$ F500, Tecan, Shanghai, China), applying $495 \mathrm{~nm}$ as the emission wavelength and $350 \mathrm{~nm}$ as the excitation wavelength. Each reaction was repeated three times in this assay to ensure reproducibility.

\subsection{Minimum Inhibitory Concentrations (MIC) and Growth Curves}

The MIC of acacetin against $S$. aureus was determined by broth microdilution on the basis of NCCLS guideline $\mathrm{M}_{31}-\mathrm{A}_{2}$. To plot the growth curves of $S$. aureus, overnight-cultured $S$. aureus was diluted by 1:100 into sterile BHI broth that contained or did not contain appropriate concentrations of acacetin. Absorbance at $600 \mathrm{~nm}$ was measured at different intervals of time.

\subsection{Fibrinogen-Binding Assay}

Overnight cultured S. aureus ATCC25904 strain was diluted 1:100 into sterile BHI broth and incubated along with variable concentrations of acacetin at $37^{\circ} \mathrm{C}$ until $A_{600}$ reached 0.5 . Positive control was the $\Delta$ SrtA strain cultured using the same conditions. Then, all of the cell suspensions were removed, deposited by centrifugation $(5000 \times g$ for $5 \mathrm{~min})$, and suspended in Phosphate buffered saline (PBS) to an $A_{600}$ of 1.0. The resuspended cells were added to the wells of a fibrinogen-coated $\left(20 \mu \mathrm{g} / \mathrm{mL}\right.$ bovine fibrinogen, overnight at $\left.4{ }^{\circ} \mathrm{C}\right)$ polystyrene Costar 96 -well plate and incubated at $37^{\circ} \mathrm{C}$ for $2 \mathrm{~h}$. The liquid was removed. Bacteria were washed with PBS, and $25 \%(v / v)$ formaldehyde was added to fix samples. Adherent bacterial cells were stained with crystal violet solution (12.5 g/L) for $10 \mathrm{~min}$, the wells were washed again with PBS and dried, and the absorbance at $570 \mathrm{~nm}$ of disparate samples was subsequently measured. The results were shown using the percentage of the tested group compared to the wild type group.

\subsection{SpA-Related Fluorescence Analysis}

Overnight cultured S. aureus was diluted by 1:100 into sterile BHI broth and incubated with or without appropriate concentrations of acacetin at $37{ }^{\circ} \mathrm{C}$ until $A_{600}$ reached 0.5 . The bacteria were washed twice with PBS and then blocked with $2 \%$ BSA for $1 \mathrm{~h}$. The bacteria were centrifuged and suspended in PBS containing a 1:100 dilution of FITC-labelled goat anti-rabbit IgG (eBioscience). 
After $1 \mathrm{~h}$ of incubation at room temperature, the S. aureus were washed twice with PBS and added to poly-L-lysine-coated glass slides. The fluorescence was observed using a confocal laser-scanning microscope (Olympus, Shanghai, China).

\subsection{Statistical Analysis}

The log-rank test (Mantel-Cox) was used to analyze the survival rate data, and the two-tailed Student's $t$-test was used to assess the significance of Fg-binding data and bacterial burden data; $p<0.05$ was considered statistically significant. The molecular dynamics and animal model experimental methods that were used are described in the Supplementary Materials section.

\section{Conclusions}

Overall, our results suggest that acacetin is a potent and promising small molecule anti-SrtA agent which prevents the surface protein Spa from anchoring in the cell wall and prevents the clumping factors (ClfA and ClfB) from playing an adhesion function. The potential therapeutic effect of acacetin on S. aureus-induced fatal infection and renal abscesses made acacetin worthy for further development into a novel therapeutic agent. We will improve its physical and chemical properties and biological activities through structural modification and other optimization measures to develop it for use as an alternative or complementary treatment of $S$. aureus infections.

Supplementary Materials: Supplementary materials can be accessed at: http:/ /www.mdpi.com/1420-3049/21/ 10/1285/s1.

Acknowledgments: This study was funded by National Nature Science Foundation of China (No. 31272608, No. 31502117) and China Postdoctoral Science Foundation funded project (2014M561301, 2015T80309).

Author Contributions: The contributions of the respective authors are as follows: Lin Wang and Dacheng Wang designed the study. Chongwei Bi and Hongjun Cai performed the in vivo experiments. Xiaoyun Dong and Xiaobo Zhong performed the in vitro experiments. Dacheng Wang performed the molecular docking and dynamic simulation experiments. Lin Wang and Chongwei Bi wrote the manuscript.

Conflicts of Interest: The authors declare no conflict of interest.

\section{References}

1. Fitzgerald-Hughes, D.; Devocelle, M.; Humphreys, H. Beyond conventional antibiotics for the future treatment of methicillin-resistant Staphylococcus aureus infections: Two novel alternatives. FEMS. Immunol. Med. Microbiol. 2012, 65, 399-412. [CrossRef] [PubMed]

2. Cascioferro, S.; Cusimano, M.G.; Schillaci, D. Antiadhesion agents against Gram-positive pathogens. Future Microbiol. 2014, 9, 1209-1220. [CrossRef] [PubMed]

3. Paterson, G.K.; Mitchell, T.J. The biology of Grampositive sortase enzymes. Trends Microbiol. 2004, 12, 89-95. [CrossRef] [PubMed]

4. Scott, J.R.; Barnett, T.C. Surface proteins of grampositive bacteria and how they get there. Ann. Rev. Microbiol. 2006, 60, 397-423. [CrossRef] [PubMed]

5. Clancy, K.W.; Melvin, J.A.; McCafferty, D.G. Sortase transpeptidases: Insights into mechanism, substrate specificity, and inhibition. Biopolymers 2010, 94, 385-396. [CrossRef] [PubMed]

6. Ton-That, H.; Marraffini, A.; Schneewind, O. Protein sorting to the cell wall envelope of Gram-positive bacteria. Biochim. Biophys. Acta 2004, 1694, 269-278. [CrossRef] [PubMed]

7. Mazmanian, S.K.; Liu, G.; Ton-That, H.; Schneewind, O. Staphylococcus aureus Sortase, an enzyme that anchors surface proteins to the cell wall. Science 1999, 285, 760-763. [CrossRef] [PubMed]

8. Spirig, T.; Weiner, E.M.; Clubb, R.T. Sortase enzymes in Gram-positive bacteria. Mol. Microbiol. 2011, 82, 1044-1059. [CrossRef] [PubMed]

9. Mazmanian, S.K.; Liu, G.; Jensen, E.R.; Lenoy, E.; Schneewind, O. Staphylococcus aureus sortase mutants defective in the display of surface proteins and in the pathogenesis of animal infections. Proc. Natl. Acad. Sci. USA 2000, 97, 5510-5515. [CrossRef] [PubMed]

10. Nandakumar, R.; Nandakumar, M.P.; Marten, M.R.; Ross, J.M. Proteome analysis of membrane and cell wall associated proteins from Staphylococcus aureus. J. Proteome Res. 2005, 4, 250-257. [CrossRef] [PubMed] 
11. Suree, N.; Jung, M.E.; Clubb, R.T. Recent advances towards new anti-infective agents that inhibit cell surface protein anchoring in Staphylococcus aureus and other gram-positive pathogens. Mini Rev. Med. Chem 2007, 7, 991-1000. [CrossRef] [PubMed]

12. Cascioferro, S.; Totsika, M.; Schillaci, D. Sortase A: An ideal target for anti-virulence drug development. Microb. Pathog. 2014, 77, 105-112. [CrossRef] [PubMed]

13. Mazmanian, S.K.; Ton-That, H.; Su, K.; Schneewind, O. An iron-regulated sortase enzyme anchors a class of surface protein during Staphylococcus aureus pathogenesis. Proc. Natl. Acad. Sci. USA 2002, 99, 2293-2298. [CrossRef] [PubMed]

14. Cheng, A.G.; Kim, H.K.; Burts, M.L.; Krausz, T.; Schneewind, O.; Missiakas, D.M. Genetic requirements for Staphylococcus aureus abscess formation and persistence in host tissues. FASEB J. 2009, 23, 3393-3404. [CrossRef] [PubMed]

15. McAdow, M.; Kim, H.K.; Dedent, A.C.; Hendrickx, A.P.; Schneewind, O.; Missiakas, D.M. Preventing Staphylococcus aureus sepsis through the inhibition of its agglutination in blood. PLoS Pathog. 2011, 7, e1002307. [CrossRef] [PubMed]

16. Wang, L.; Bi, C.; Wang, T.; Xiang, H.; Chen, F.; Hu, J.; Liu, B.; Cai, H.; Zhong, X.; Deng, X.; et al. Coagulase-negative and nonhemolytic strain of Staphylococcus aureus for investigating the roles of SrtA in S. aureus-induced bloodstream infection. Pathog. Dis. 2015. [CrossRef] [PubMed]

17. Cascioferro, S.; Raffa, D.; Maggio, B.; Raimondi, M.V.; Schillaci, D.; Daidone, G. Sortase A inhibitors: Recent advances and future perspectives. J. Med. Chem. 2015, 58, 9108-9123. [CrossRef] [PubMed]

18. Cossart, P.; Jonquieres, R. Sortase, a universal target for therapeutic agents against gram-positive bacteria? Proc. Natl. Acad. Sci. USA 2000, 97, 5013-5015. [CrossRef] [PubMed]

19. Maresso, A.W.; Schneewind, O. Sortase as a target of anti-infective therapy. Pharmacol. Rev. 2008, 60, $128-141$. [CrossRef] [PubMed]

20. Liu, B.; Chen, F.; Bi, C.; Wang, L.; Zhong, X.; Cai, H.; Deng, X.; Niu, X.; Wang, D. Quercitrin, an inhibitor of Sortase A, interferes with the adhesion of Staphylococcal aureus. Molecules 2015, 20, 6533-6543. [CrossRef] [PubMed]

21. Wang, L.; Bi, C.; Cai, H.; Liu, B.; Zhong, X.; Deng, X.; Wang, T.; Xiang, H.; Niu, X.; Wang, D. The therapeutic effect of chlorogenic acid against Staphylococcus aureus infection through sortase A inhibition. Front. Microbiol. 2015, 6, 1031. [CrossRef] [PubMed]

22. Fan, L.H.; Li, X.; Chen, D.Y.; Zhang, N.; Wang, Y.; Shan, Y.; Hu, Y.; Xu, R.A.; Jin, J.; Ge, R.S. Determination of acacetin in rat plasma by UPLC-MS/MS and its application to a pharmacokinetic study. J. Chromatogr. B 2015, 986-987, 18-22. [CrossRef] [PubMed]

23. Valkama, E.; Salminen, J.P.; Koricheva, J.; Pihlaja, K. Changes in leaf trichomes and epicuticular flavonoids during leaf development in three birch taxa. Ann. Bot. 2004, 94, 233-242. [CrossRef] [PubMed]

24. Bhat, T.A.; Nambiar, D.; Tailor, D.; Pal, A.; Agarwal, R.; Singh, R.P. Acacetin inhibits in vitro and in vivo angiogenesis and downregulates Stat signaling and VEGF expression. Cancer Prev. Res. 2013, 6, 1128-1139. [CrossRef] [PubMed]

25. Chien, S.T.; Lin, S.S.; Wang, C.K.; Lee, Y.B.; Chen, K.S.; Fong, Y.; Shih, Y.W. Acacetin inhibits the invasion and migration of human non-small cell lung cancer A549 cells by suppressing the p38alpha MAPK signaling pathway. Mol. Cell Biochem. 2011, 350, 135-148. [CrossRef] [PubMed]

26. Shen, K.H.; Hung, S.H.; Yin, L.T.; Huang, C.S.; Chao, C.H.; Liu, C.L.; Shih, Y.W. Acacetin, a flavonoid, inhibits the invasion and migration of human prostate cancer DU145 cells via inactivation of the p38 MAPK signaling pathway. Mol. Cell Biochem. 2010, 333, 279-291. [CrossRef] [PubMed]

27. Watanabe, K.; Kanno, S.; Tomizawa, A.; Yomogida, S.; Ishikawa, M. Acacetin induces apoptosis in human T cell leukemia Jurkat cells via activation of a caspase cascade. Oncol. Rep. 2012, 27, 204-209. [PubMed]

28. Chen, W.P.; Yang, Z.G.; Hu, P.F.; Bao, J.P.; Wu, L.D. Acacetin inhibits expression of matrix metalloproteinases via a MAPK-dependent mechanism in fibroblast-like synoviocytes. J. Cell Mol. Med. 2015, 19, 1910-1915. [CrossRef] [PubMed]

29. Komape, N.P.; Aderogba, M.; Bagla, V.P.; Masoko, P.; Eloff, J.N. Anti-bacterial and anti-oxidant activities of leaf extracts of Combretum vendae (Combretecacea) and the isolation of an anti-bacterial compound. Afr. J. Tradit. Complement. Altern. Med. 2014, 11, 73-77. [CrossRef] [PubMed] 
30. Ton-That, H.; Liu, G.; Mazmanian, S.K.; Faull, K.F.; Schneewind, O. Purification and characterization of sortase, the transpeptidase that cleaves surface proteins of Staphylococcus aureus at the LPXTG motif. Proc. Natl. Acad. Sci. USA 1999, 96, 12424-12429. [CrossRef] [PubMed]

31. Lee, J.C.; Betley, M.J.; Hopkins, C.A.; Perez, N.E.; Pier, G.B. Virulence studies, in mice, of transposon-induced mutants of Staphylococcus aureus differing in capsule size. J. Infect. Dis. 1987, 156, 741-750. [CrossRef] [PubMed]

32. Lowy, F.D. Staphylococcus aureus infections. N. Engl. J. Med. 1998, 339, 520-532. [CrossRef] [PubMed]

33. Ippolito, G.; Leone, S.; Lauria, F.N.; Nicastri, E.; Wenzel, R.P. Methicillin-resistant Staphylococcus aureus: The superbug. Int. J. Infect. Dis. 2010, 14, S7-S11. [CrossRef] [PubMed]

34. Oh, K.B.; Nam, K.W.; Ahn, H.; Shin, J.; Kim, S.; Mar, W. Therapeutic effect of (Z)-3-(2,5-dimethoxyphenyl)2-(4-methoxyphenyl) acrylonitrile (DMMA) against Staphylococcus aureus infection in a murine model. Biochem. Biophys. Res. Commun. 2010, 396, 440-444. [CrossRef] [PubMed]

35. Zhang, J.; Liu, H.; Zhu, K.; Gong, S.; Dramsi, S.; Wang, Y.T.; Li, J.; Chen, F.; Zhang, R.; Zhou, L.; et al. Antiinfective therapy with a small molecule inhibitor of Staphylococcus aureus sortase. Proc. Natl. Acad. Sci. USA 2014, 111, 13517-13522. [CrossRef] [PubMed]

36. Chen, F.; Liu, B.; Wang, D.; Wang, L.; Deng, X.; Bi, C.; Xiong, Y.; Wu, Q.; Cui, Y.; Zhang, Y.; et al. Role of sortase A in the pathogenesis of Staphylococcus aureus-induced mastitis in mice. FEMS Microbiol. Lett. 2014, 351, 95-103. [CrossRef] [PubMed]

Sample Availability: Samples of the compounds screened in this study are available from the authors.

(C) 2016 by the authors; licensee MDPI, Basel, Switzerland. This article is an open access article distributed under the terms and conditions of the Creative Commons Attribution (CC-BY) license (http://creativecommons.org/licenses/by/4.0/). 